\title{
Noise immune dielectric modulated dual trench transparent gate engineered MOSFET as a label free biosensor: proposal and investigation
}

\author{
Dipanjan Sen ${ }^{1}\left[\right.$ Arpan De $^{1} \cdot$ Bijoy Goswami ${ }^{1} \cdot$ Sharmistha Shee $^{1} \cdot$ Subir Kumar Sarkar $^{1}$
}

Received: 5 January 2021 / Accepted: 10 September 2021 / Published online: 30 September 2021

(c) The Author(s), under exclusive licence to Springer Science+Business Media, LLC, part of Springer Nature 2021

\begin{abstract}
We propose and investigate a biosensor based on a transparent dielectric-modulated dual-trench gate-engineered metaloxide-semiconductor field-effect transistor (DM DT GE-MOSFET) for label-free detection of biomolecules with enhanced sensitivity and efficiency. Various sensing parameters such as the $I_{\mathrm{ON}} / I_{\mathrm{OFF}}$ ratio and the threshold voltage shift are evaluated as metrics to validate the proposed sensing device. Additionally, $S_{V \text { th }}$ (the $V_{\text {th }}$ sensitivity) is also analyzed, considering both positively and negatively charged biomolecules. In addition, radiofrequency (RF) sensing parameters such as the transconductance gain and the cutoff frequency are taken into account to provide further insight into the sensitivity of the proposed device. Furthermore, the linearity, distortion, and noise immunity of the device are evaluated to confirm the overall performance of the biosensor at high $(\mathrm{GHz})$ frequency. The results indicate that the proposed biosensor exhibits a $S_{V \mathrm{th}}$ value of 0.68 for positively charged biomolecules at a very low drain bias of $0.2 \mathrm{~V}$. The proposed device can thus be used as an alternative to conventional FET-based biosensors.
\end{abstract}

Keywords Transparent gate $\cdot$ Dual-trench cavity $\cdot$ Inner gate $\cdot$ Dielectric modulation $\cdot$ Biosensor $\cdot$ Noise assessment

\section{Introduction}

The detection and accurate analysis of nanoscale biomolecules is becoming an everyday requirement due to several recent findings in the field of microbiology. The identification of hundreds of proteins, such as DNA, biotin-streptavidin, and S-protein, as well as different kinds of bacteria and viruses is crucial to understand any type of abnormal behavior inside living cells. In addition, the pathological

Dipanjan Sen

sendipanjan10@gmail.com

Arpan De

arpantukan@gmail.com

Bijoy Goswami

bijoy.ete@aec.ac.in

Sharmistha Shee

sharmi.shee@gmail.com

Subir Kumar Sarkar

sksarkar.etce@ jadavpuruniversity.in

1 Department of Electronics and Telecommunication Engineering, Jadavpur University, Kolkata, India results related to coronavirus disease 2019 (COVID-19) are analogous to those reported previously in the severe acute respiratory syndrome (SARS) and Middle East respiratory syndrome (MERS) pandemics, and biopsy samples of lung tissues depict lesions in bilateral diffuse alveolar with cellular fibromyxoid exudates. The importance of designing more efficient, highly sensitive, and low-cost biosensors has thus increased drastically.

Among all biosensors, those based on field-effect transistor (FETs) are of great research interest [1], offering several advantages such as complementary metal-oxide-semiconductor (CMOS) compatibility, high scalability, label-free detection, and low-cost production. These features make them promising candidates for use in future biosensor applications [2, 3]. FET-based biosensors have significant application potential for label-free detection of charged bioanalytes [4-7]. The formation of a vertical nanogap with the biosensor enables the detection of charge-free molecules as well [7-12]. The modulation of the coupling between the gate and channel based on different charged/ uncharged bioanalytes with a wide range of permittivity is the operating principle of dielectric-modulated FET-based biosensors [13]. To cite a few examples, (3-aminopropyl) 
triethoxysilane (APTES) $(K=3.57)$, streptavidin $(K=2.1)$, biotin $(K=2.63)$ [14], food proteins such as gluten $(K=5)$, keratin $(K=10)$, zenin $(K=7)$, and gelatin $(K=12)$ [15] have different permittivity values but are uncharged in nature, whereas example charged bioanalytes include amino acids such as glutamic acid, aspartic acid, arginine, lysine, and histidine $(K=11-25)$ [16]. Moreover, the S-protein of the SARS-CoV-2 virus usually has glycoproteins in it, and the dielectric constant of the protein lies in the range of 1-4 [17], similar to the values for biotin or streptavidin. All these biomolecules have been successfully detected by previously reported FET-based biosensors. With a focus on dielectricmodulated biosensing applications, split-gate JL MOSFETs [18], gate-underlap DG MOSFET [19, 20], and surroundinggate MOSFET [21] have been reported.

We propose herein a noise-immune dielectric-modulated dual-trench MOSFET (DM DT GE-MOSFET)-based biosensor with a split transparent indium-doped tin oxide (ITO) outer gate structure coupled with an inner gate. This architecture provides better control over the channel profile through both the exterior and interior gate arrangements. The inclusion of the inner gate enhances the device performance by forming an additional inversion layer. Its improved drain current and decent threshold voltage shift make this device suitable for use in low-power biosensing applications. The biosensing capabilities of the device are exhibited for various bioanalytes, including DNA, proteins, etc. as notable example. The tied-gate symmetric arrangement with dielectric modulation forms the operating principle of the structure proposed herein. The cavity is created on the top and bottom of the device with split gates in between, forming a trench-like architecture. Furthermore, the device shows impressive results in terms of RF sensing metrics and noise performance.

\section{The device structure, simulation framework, and calibration methodology}

A three-dimensional (3D) schematic and corresponding twodimensional (2D) projection (top view) diagram as well as the possible fabrication steps for the proposed device are shown in Fig. 1a-b. Here, the proposed device has a dualtrench split-gate architecture with an additional "inner" gate (IG) at the source site. The thickness of the outer gates on the top and bottom is $2 \mathrm{~nm}$, while that of the IG is $1 \mathrm{~nm}$, being buried inside the silicon film. The channel length $\left(L_{\mathrm{C}}\right)$ is $50 \mathrm{~nm}$, whereas the source length $\left(L_{\mathrm{S}}\right)$ and drain length $\left(L_{\mathrm{D}}\right)$ are 20 and $30 \mathrm{~nm}$, respectively. A nanocavity is embedded at the top and bottom of the device with intermediate positioning of the control gates, having a length of $15 \mathrm{~nm}$. Moreover, the maximum height of the biosensor cavity $\left(T_{\text {CAVITY }}\right)$ is $4 \mathrm{~nm}$. For immobilization of biomolecules within the cavity, a layer of oxide film is included. The oxide layer is formed by exposure of the silicon surface to the outer atmosphere, and the thickness of $\mathrm{SiO}_{2}\left(T_{\mathrm{OX}}\right)$ to be grown is considered to be $1 \mathrm{~nm}$ for this analysis. Here, $\mathrm{a} \mathrm{SiO}_{2}$ layer is used, outlining the inner gate (IG) to avoid direct connection with the silicon film. The same external bias is applied to all the gates in the biosensing analysis. The length of the gates used for the top and bottom parts is $20 \mathrm{~nm}$, whereas the length of the inner gate is $25 \mathrm{~nm}$. The source and drain regions are highly doped with $n^{+}$dopants $\left(10^{19} \mathrm{~cm}^{-3}\right)$, while the channel is lightly doped $p$-type silicon, almost intrinsic. In addition, ITO with a work function of $4.7 \mathrm{eV}$ is used as the gate metal to enhance the current in the proposed device. Also, a gate bias $\left(V_{\mathrm{GS}}\right)$ of $0.7 \mathrm{~V}$ and a drain bias $\left(V_{\mathrm{DS}}\right)$ of $0.2 \mathrm{~V}$ are considered throughout the analysis of the proposed device. Furthermore, quantum confinement issues are bound to affect nanoscale devices, but they do not exhibit a significant effect in this case as the thickness of the silicon film is greater than $7 \mathrm{~nm}$. Hence, they are not included in this work.

In this work, the SILVACO ATLAS technology computer-aided design (TCAD) tool [22] is used as a potential candidate method to extract the simulation results and values of the figure of merits. Several physical models are applied, including the Shockley-Read-Hall model [22] for carrier recombination and generation. Lombardi's CVT model is considered to include the mobilities due to carrier scattering caused by the parallel and perpendicular electric fields applied to the device. Also, the bandgap narrowing or BGN model is also used here. The Fermi-Dirac distribution or statistical model is used to increase the efficacy. Additionally, to include information related to the energy (temperature) of the charge carriers, the energy balance transport (EBT) model [22] is added. The physical models applied in the simulation setup are calibrated against experimental results [23] as shown in Fig. 2.

\section{Results and discussion}

\subsection{The (lateral and vertical) electron concentration profile of the proposed device}

The electron concentration profile of the device for different biomolecules is shown in Fig. 3a, b. It can be seen that, as the dielectric constant of the biomolecule in the cavity is increased, the electron concentration in the channel also increases. This behavior can be attributed to the increase in the electric field across the oxide layer due to the higher permittivity, which causes more electrons to emerge from the bulk and consequently increases the electron concentration in the channel (Fig. 3a). Moreover, Fig. $3 \mathrm{~b}$ exhibits the same trend in the vertical direction, as can be seen along the channel. Here, the presence of the 

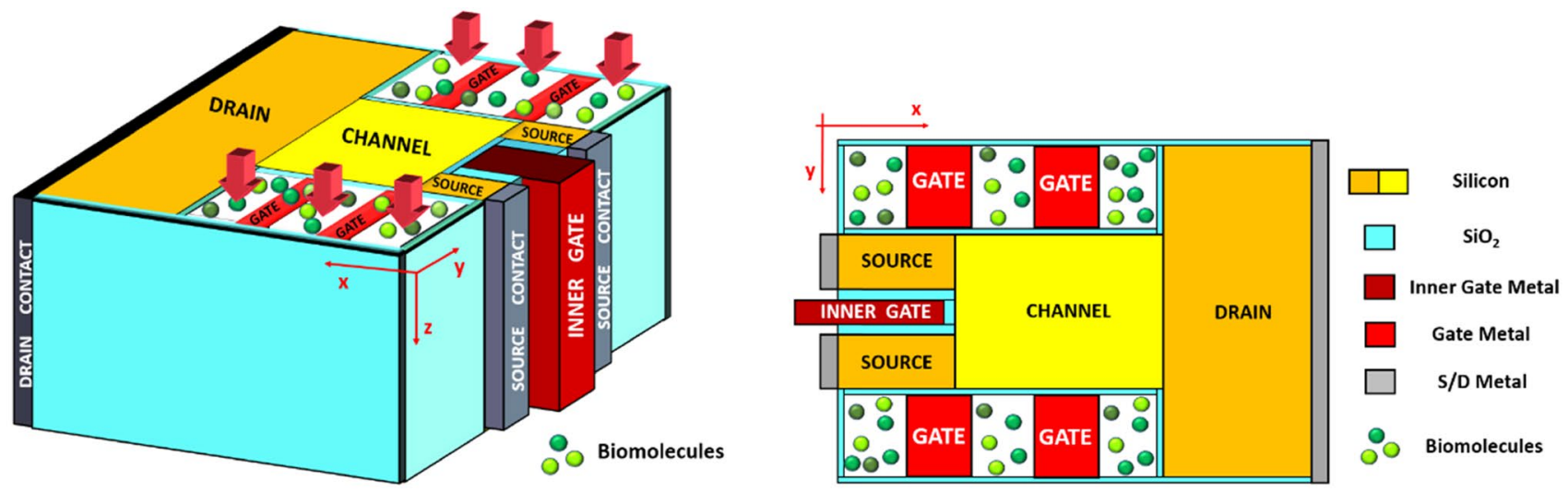

(a) 3D Schematic of the proposed FET-Biosensor (Left) and 2D top-view projection (Right) of the 3D a rchitecture shown to the left.

\section{Process Flow}

(a) MBE Growth of p-type Si followed by Source/Drain Doping
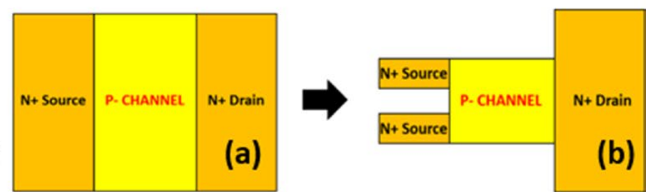

(b) Trench Creation for Gate (Inner \& Outer) and Cavity Accommodation

(c) $\mathrm{SiO}_{2}$ Deposition

(d) Selective etching of $\mathrm{SiO}_{2}$ for Inner and Outer Gate Formation

(e) LPCVD of ITO and Patterning for Gate/Source/Drain Contacts

(f) Etching to create nano cavity for Biomolecule
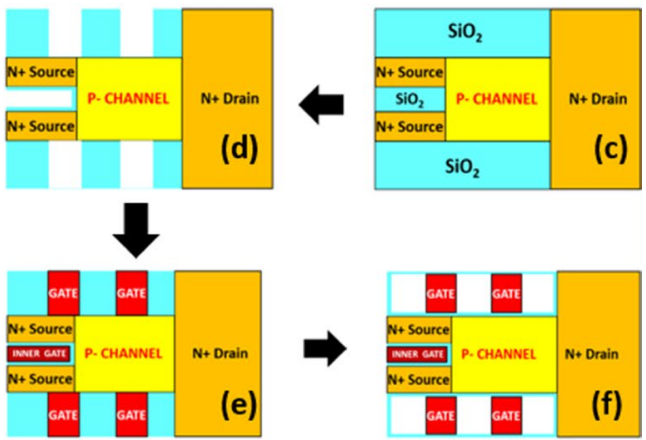
Accommodation

(g) Final Structure

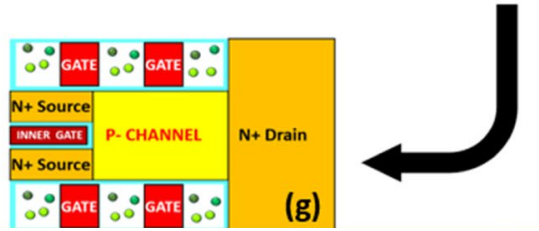

(b) Fabrication Process Flow of the proposed architecture

Fig. 1 a A 3D schematic of the proposed FET-based biosensor (Left) and a 2D top-view projection (right) of the 3D architecture shown to the left. b A potential fabrication process flow for the proposed architecture

inner gate at the source-channel interface helps to create a sufficient inversion layer throughout the channel region, thereby reducing the on-resistance of the device. Therefore, the electron concentration profile of the proposed device reveals that the inclusion of the extra inner gate at the source region forms a second inversion layer and thus reduces the channel resistance drastically.

\subsection{The surface potential characteristics of the proposed device}

Figure 4 shows the surface potential of the DM DT GEMOSFET-based biosensor in the presence of different biomolecules. Moreover, immobilization of the biomolecules inside the nanocavity results in a change in the potential due 


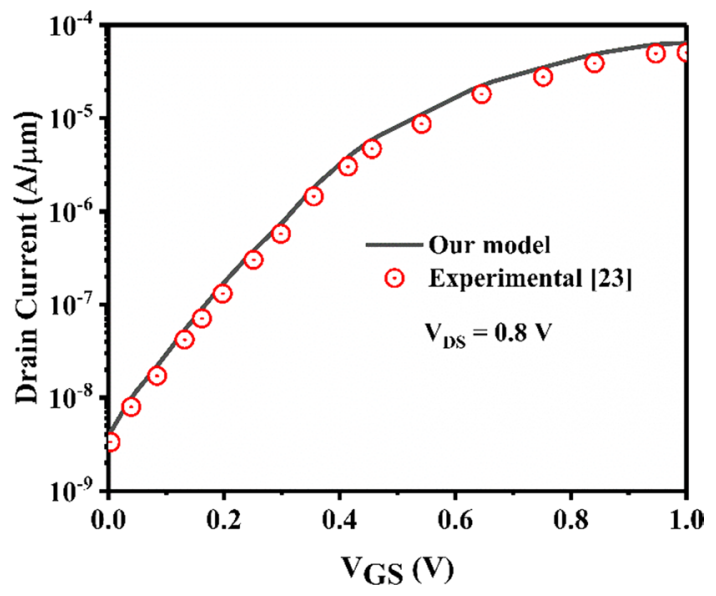

Fig. 2 The calibration of the simulation models against the experimental data reported in Ref. [23]

to the their different dielectric constant values. Furthermore, this shift in the surface potential can be used as a sensing metric to detect different biomolecules trapped inside the nanocavity region.

\subsection{The drain current and the $I_{\mathrm{ON}} / I_{\mathrm{OFF}}$ characteristics of the proposed device:}

Figure 5a depicts the variation of the drain current with the applied gate bias for different dielectric constants at a constant drain bias of $0.2 \mathrm{~V}$. Note that the increase of the $I_{\mathrm{ON}} / I_{\mathrm{OFF}}$ ratio with the variation of the dielectric constants of the different biomolecules can be used as a potential sensing metric, as shown in Fig. 5b. Here, the effective change in the $I_{\mathrm{ON}} / I_{\mathrm{OFF}}$ ratio of the device from $K=1\left(\sim 7 \times 10^{4}\right)$ to $\mathrm{K}=10\left(\sim 10^{8}\right)$ is more than three orders at $V_{\mathrm{GS}}=0.7 \mathrm{~V}$ and
$V_{\mathrm{DS}}=0.2 \mathrm{~V}$. Thus, the enhancement in the switching ratio for a particular biomolecule can be used as a key parameter for its detection/sensing. As seen in Fig. $5 \mathrm{a}, I_{\mathrm{OFF}}$ changes by an order of $\sim 2.5$-fold when the dielectric constant of the biomolecule changes from 1 to 10 . Therefore, the proposed device shows impressive performance in terms of the current sensitivity metric at an ultralow supply voltage of $0.2 \mathrm{~V}$.

\subsection{The threshold voltage and its sensitivity $\left(S_{v \text { th }}\right)$ profile for the proposed device}

Figure 6a shows the threshold voltage profile when applying different biomolecules. It is quite evident that the threshold voltage of the device increases with an increase of the dielectric constant of the biomolecule. Here, the sensitivity of the DM DT GE-MOSFET-based biosensor in the presence

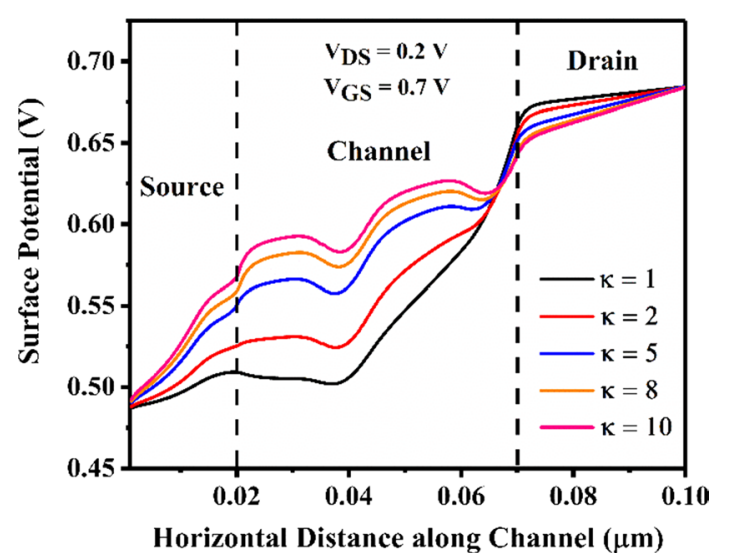

Fig.4 The surface potential profile of the DM DT GE-MOSFETbased biosensor

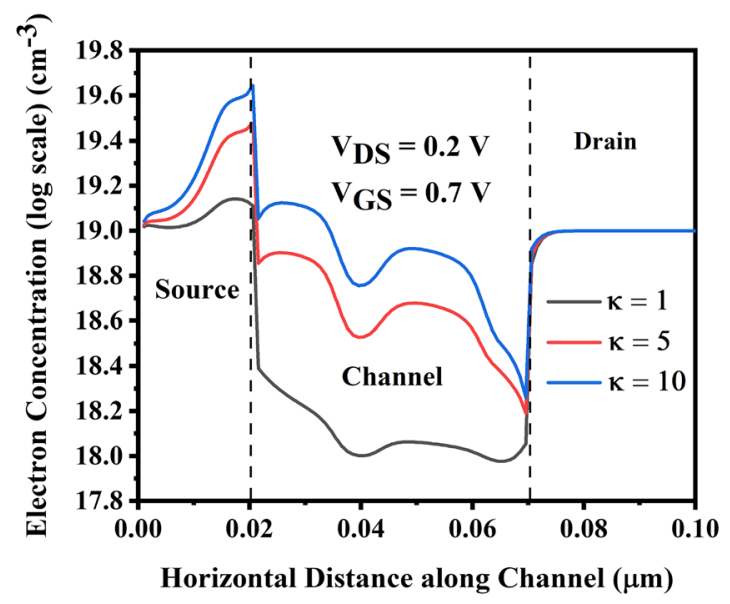

(a)

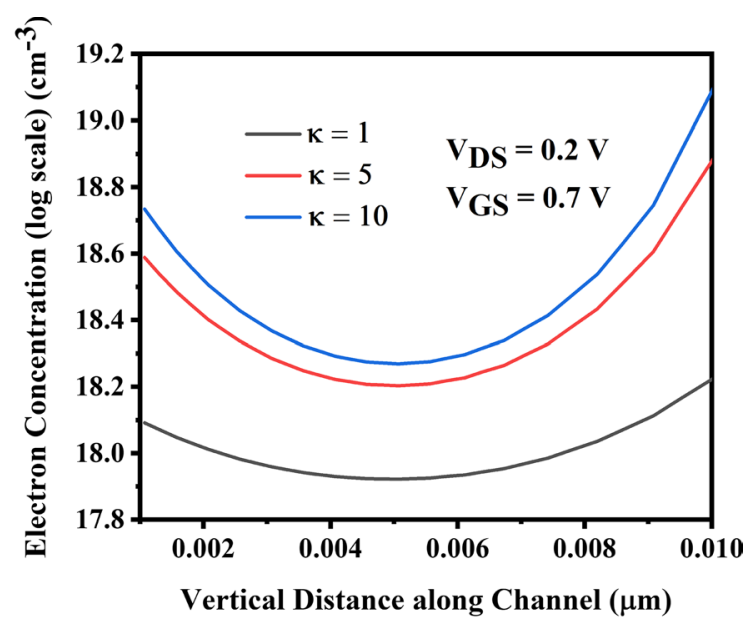

(b)

Fig. 3 The electron concentration profile of the DM DT GE-MOSFET-based biosensor in the a lateral and b vertical direction 


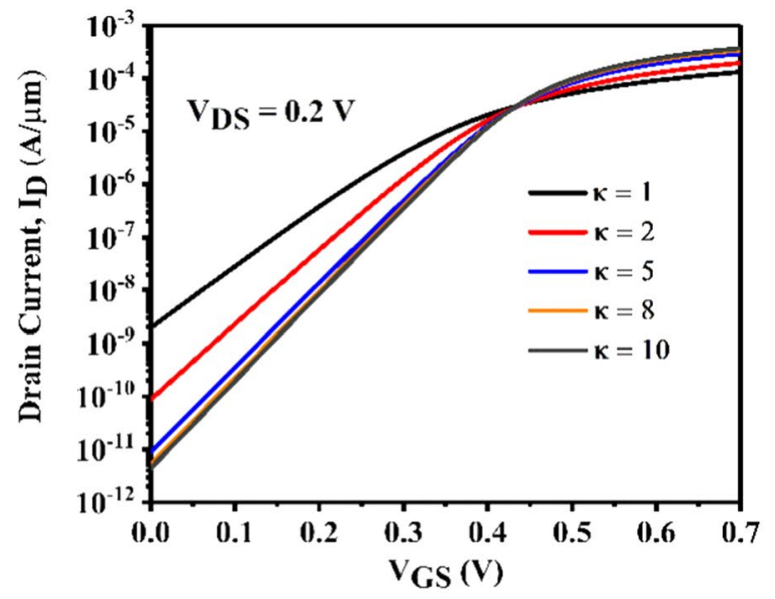

(a)

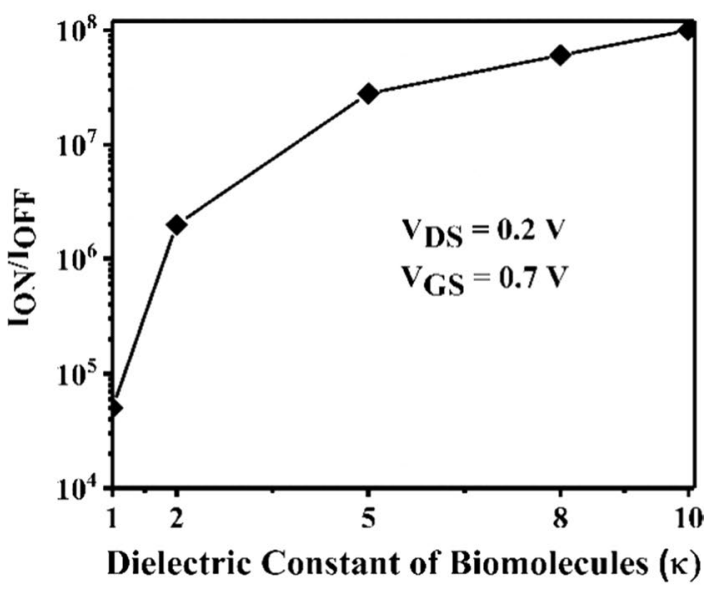

(b)

Fig. 5 a The drain current versus the applied gate bias. b The $I_{\mathrm{ON}} / I_{\mathrm{OFF}}$ ratio for different permittivity values of a wide range of biomolecules

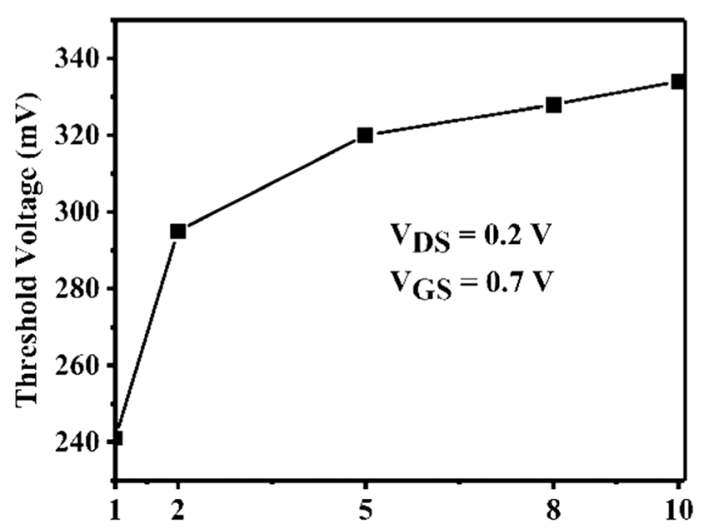

Dielectric Constant of Biomolecules $(\kappa)$

(a)

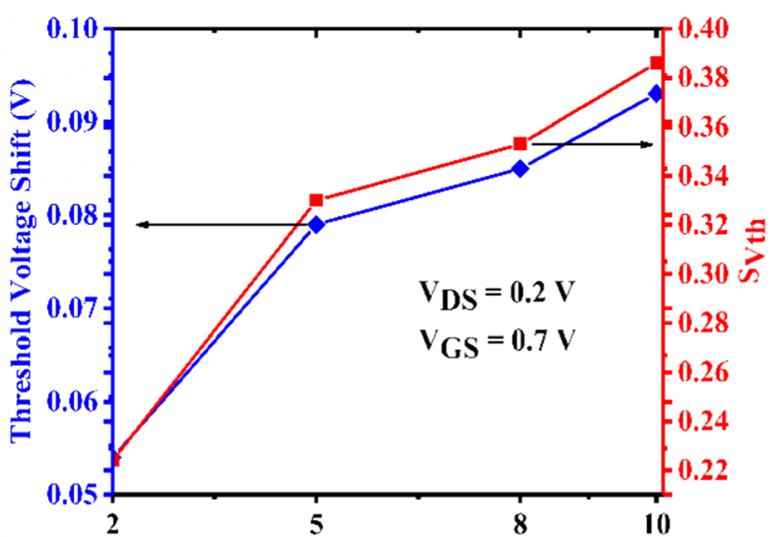

Dielectric Constant of Biomolecules (к)

(b)

Fig. 6 a The threshold voltage and b its sensitivity $\left(S_{V \text { th }}\right)$ characteristics for the DM DT GE-MOSFET-based biosensor with neutral biomolecules

of different biomolecules in the nanocavity can be defined in terms of the shift in the threshold voltage as:

$S_{V_{\mathrm{th}}}=\left|\frac{V_{\mathrm{th}}(\text { air })-V_{\mathrm{th}}(\text { bio })}{V_{\mathrm{th}}(\text { air })}\right|$,

where $V_{\text {th }}$ (air) and $V_{\text {th }}$ (bio) are the threshold voltages of the DM DT GE-MOSFET (measured with the help of constant-current method at $\left.I_{\mathrm{D}}=10^{-7} \mathrm{~A} / \mu \mathrm{m}\right)$ in the absence of biomolecules in the nanocavity (air filled) and with biomolecules present in the nanocavity, respectively. The maximum change of the threshold voltage is nearly $100 \mathrm{mV}$ at $K=10$ (when filled with keratin) while the minimum change is $52 \mathrm{mV}$ at $K=2$ (when filled with streptavidin), as shown in Fig. 6b. Furthermore, the relative change in the $V_{\text {th }}$ shift values for keratin and streptavidin is $\sim 92.3 \%$, indicating that the proposed device is highly sensitive for detecting specific biomolecules. Additionally, the absolute sensitivity profile shown in Fig. 6b depicts that the proposed device is capable of detecting biomolecules at a very low supply voltage and is thus suitable for low-power sensing applications.

\subsection{The threshold voltage sensitivity $\left(S_{v t h}\right)$ for positively and negatively charged biomolecules}

In addition, DM MOSFET-based biosensors can also exhibit high sensitivity to charged biomolecules, although the device type ( $n$ - or $p$-type MOSFET) must be changed to effectively capture the different polarity of the charge depending on 
the ionized biomolecule of interest [24]. These charges are generally localized at the surface of the gate dielectric layer and modulate the surface potential of the channel region.

Figure 7a, b depicts the effect of charged biomolecules on the sensitivity $\left(S_{V \mathrm{th}}\right)$ of the DM DT GE-MOSFET-based biosensor. Note that positively charged biomolecules generally increase the surface potential, leading to an increased $V_{\text {th }}$ shift and a consequent reduction in the threshold voltage. Similarly, negatively charged biomolecules result in an increased threshold voltage and a reduced $V_{\text {th }}$ shift. Therefore, $S_{V t h}$ increases with the charge density of positively charged biomolecules at the $\mathrm{SiO}_{2}$ surface in the DM DT GE-MOSFET. The DM DT GE-MOSFET exhibits a significantly high sensitivity $\left(S_{V \mathrm{th}}\right)$ of 0.67 when the nanocavity is filled with keratin $(K=10)$ at a positive charge density of $10^{12} \mathrm{C} / \mathrm{cm}^{2}$, as shown in Fig. $7 \mathrm{~b}$. However, the $S_{V \mathrm{th}}$ of the device degrades in the presence of negatively charged biomolecules inside the nanocavity at a $V_{\mathrm{DS}}$ value of $0.2 \mathrm{~V}$, as shown in Fig. 7 a.

\subsection{The sensitivity of the RF characteristics of the device}

Here, the performance assessment of the proposed device is extended from an investigation of the direct-current (DC) sensing metrics to include an analysis of RF sensing metrics. The cutoff frequency $\left(f_{T}\right)$ and transconductance $\left(g_{\mathrm{m}}\right)$ are the two essential parameters for investigating the RF performance of any biosensor device. Figure 8a shows an increment in $g_{\mathrm{m}}$ with increasing dielectric constant of the biomolecules, reaching the highest gain of $1.5 \times 10^{-3} \mathrm{~S} / \mu \mathrm{m}$ at a gate bias of $0.6 \mathrm{~V}$ in the case where the cavity is filled with keratin $(K=10)$. Moreover, the proposed device exhibits a maximum sensitivity of 270 in terms of $g_{\mathrm{m}}$ at $K=10$, as shown in Fig. 8b. Also, the relative change in the sensitivity between $K=2$ and 10 is nearly $267 \%$. In addition, the cutoff frequency $f_{T}$ can be considered to be the frequency at which the current gain is unity in the small-signal model for a common-source configuration. Thus, $f_{T}$ can be written as

$f_{T}=\frac{g_{\mathrm{m}}}{2 \pi\left(C_{\mathrm{gd}}+C_{\mathrm{gs}}\right)}$.

Figure $8 \mathrm{c}$ illustrates the change in $f_{T}$ for different biomolecules against the variation of the gate bias. Note here that $f_{T}$ decreases with an increase of the dielectric constant of the biomolecule. When biomolecules with a higher dielectric constant such as keratin $(K=10)$ are immobilized inside the cavity region, the capacitive effect increases and $f_{T}$ reduces in accordance with Eq. (2). Moreover, the sensitivity in terms of $f_{T}$ increases by $211 \%$ from $K=2$ to 10 , as shown in Fig. 8d.

\subsection{An assessment of the linearity and distortion profile of the proposed device}

Furthermore, the linearity and intermodulation distortion (IMD) profile are pivotal parameters to analyze for MOSFET-based biosensors. To achieve FET-based biosensors with higher speed and improved sensitivity, less distortion and improved linearity are required. Besides, nonlinearity introduces IMD and generates an undesired signal distortion that results in a degradation of the device performance. Additionally, the parameters used to analyze biosensor performance in terms of its nonlinearity are gm 3 (the higherorder transconductance coefficient), the third-order voltage intercept point (VIP3), the third-order current intercept point

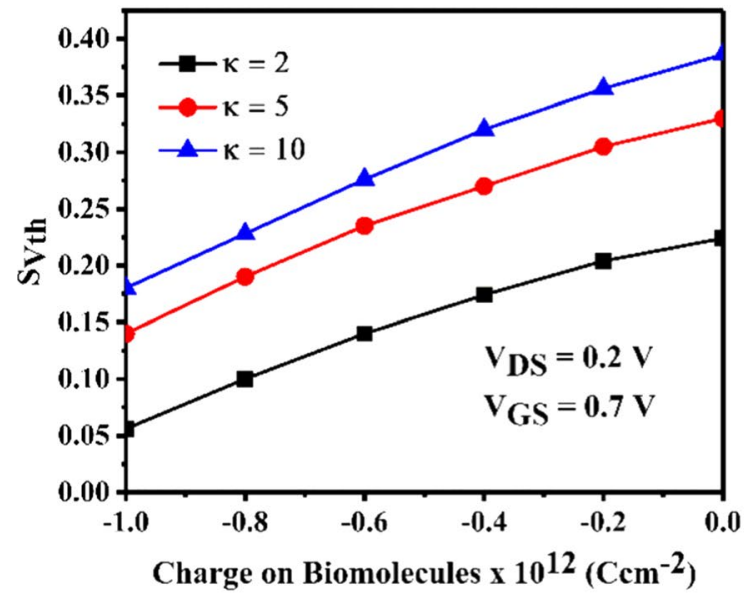

(a)

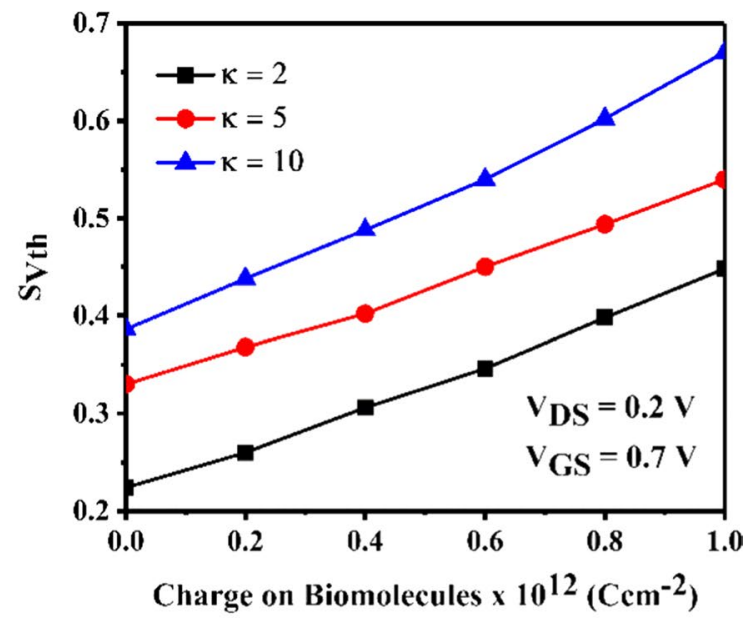

(b)

Fig. 7 The sensitivity in terms of the threshold voltage shift for a negatively charged and $\mathbf{b}$ positively charged bioanalytes 


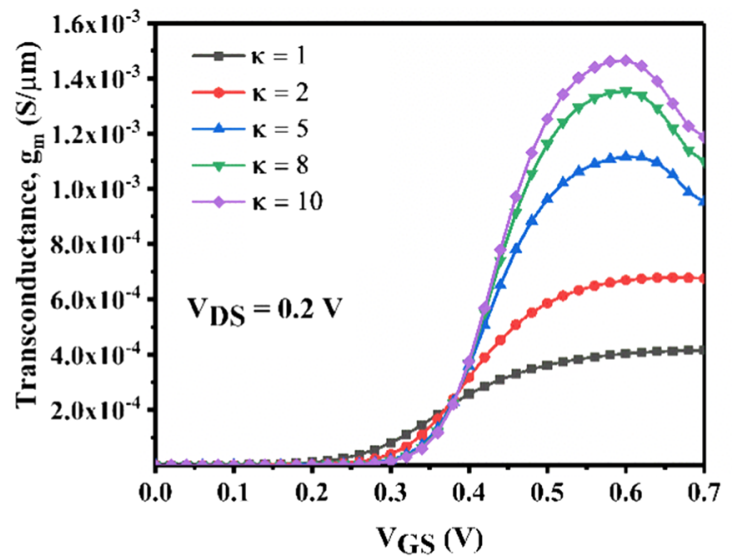

(a)

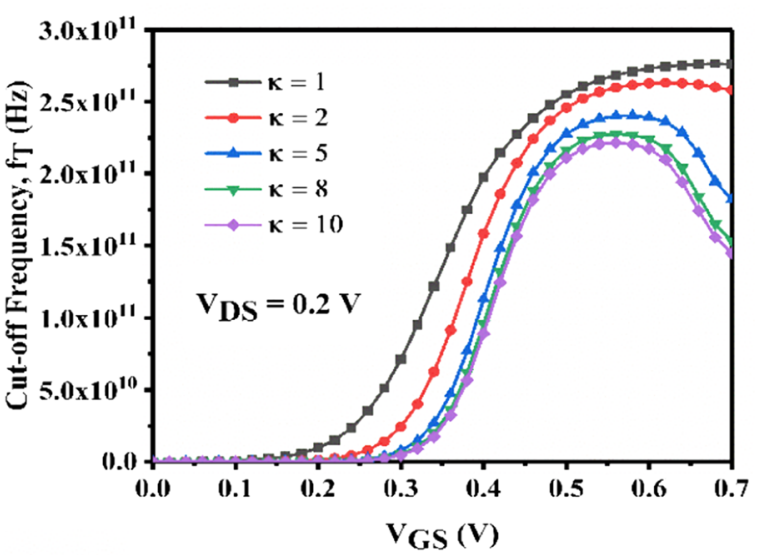

(c)

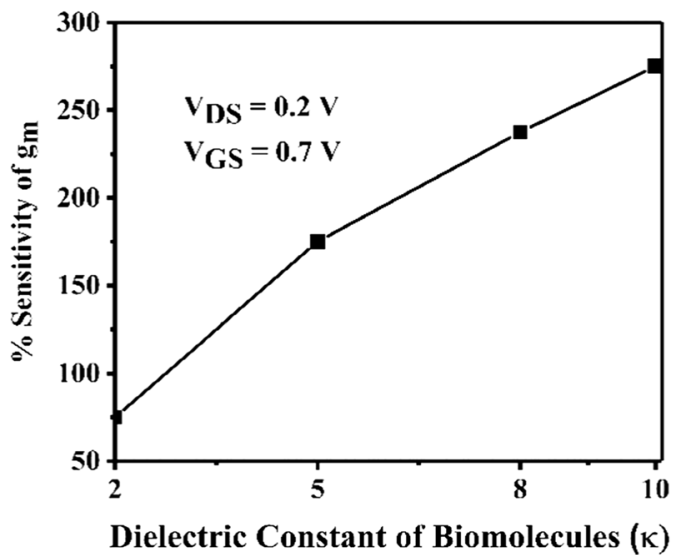

(b)

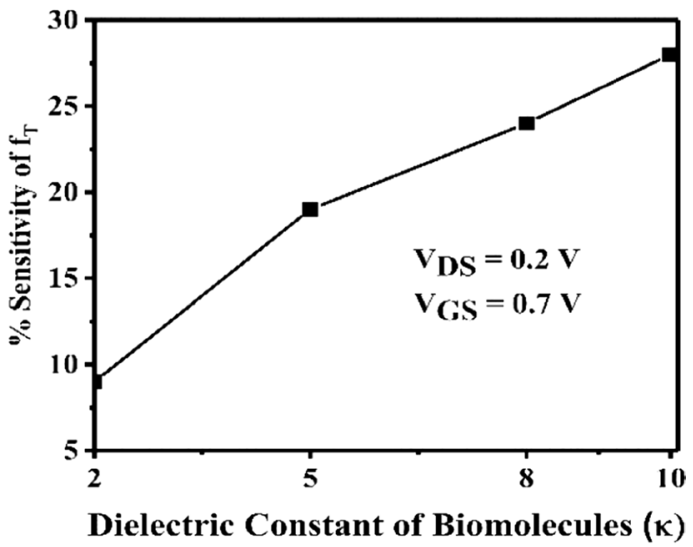

(d)

Fig. $8 \mathbf{a} g_{\mathrm{m}}$ and $\mathbf{b}$ its percentage sensitivity, and $\mathbf{c} f_{\mathrm{T}}$ and $\mathbf{d}$ its percentage sensitivity for the proposed device in the presence of different biomolecules

(IIP3), and the third-order IMD (IMD3) [25]. To achieve linear behavior, the higher-order transconductance coefficient gm3 must be as small as possible, as it limits the distortion level of the device and consequently fixes the DC bias point for optimal device performance. Figure 9 a shows that gm3 has a peak value of $0.11 \mathrm{~A} / \mathrm{V}^{3}$ and the overall value lies in the range of -0.09 to $0.11 \mathrm{~A} / \mathrm{V}^{3}$. VIP3 signifies the extrapolated voltage, and its peak value should be as high as possible to achieve less distortion and good linearity [25]. The variation of VIP3 with the input $V_{\mathrm{GS}}$ is shown in Fig. 9b. The peak of the VIP3 curve is about $1.4 \mathrm{~V}$. Moreover, the point at which the input signal and the third-order distortion signal amplitude converge is denoted as IIP3.

Like VIP3, IIP3 should also have a high peak value. Figure $9 \mathrm{c}$ depicts the IIP3 profile, revealing a peak of approximately $0.00125 \mathrm{~dB}$ at a gate bias of $0.65 \mathrm{~V}$. Furthermore, another critical parameter to analyze device reliability is IMD3, which indicates the impact of nonlinearity as a potential issue for linear amplifiers. The intermodulation current at which the first- and third-order intermodulation harmonic currents are the same is denoted as IMD3. The IMD3 profile is shown in Fig. 9d, revealing a peak value of 2.25 dB.

\subsection{An analysis of the noise immunity of the proposed device}

Figure 10a-c shows the noise characteristics of the proposed device. In addition, the presence of any external element or coupling circuit can sometimes lead to a nonrandom type of noise, which will have a detrimental effect on the performance of the biosensor. However, the effect of random noise is considered here to evaluate the electrical behavior of the device in the presence of biomolecules. Therefore, to investigate the noise immunity of the DM DT GE-MOSFET-based biosensor, noise parameters such as the minimum noise figure, the noise conductance, the output source impedance, etc. must be analyzed in depth [26]. Moreover, when a biomolecule is trapped and immobilized inside the nanocavity, all the noise figures of merit (FOMs) seem to improve at high frequency. The channel material mostly determines the channel-induced 


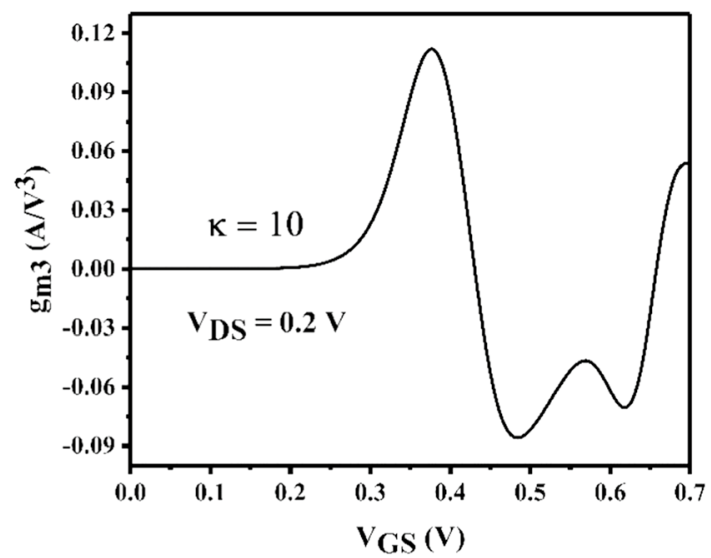

(a)

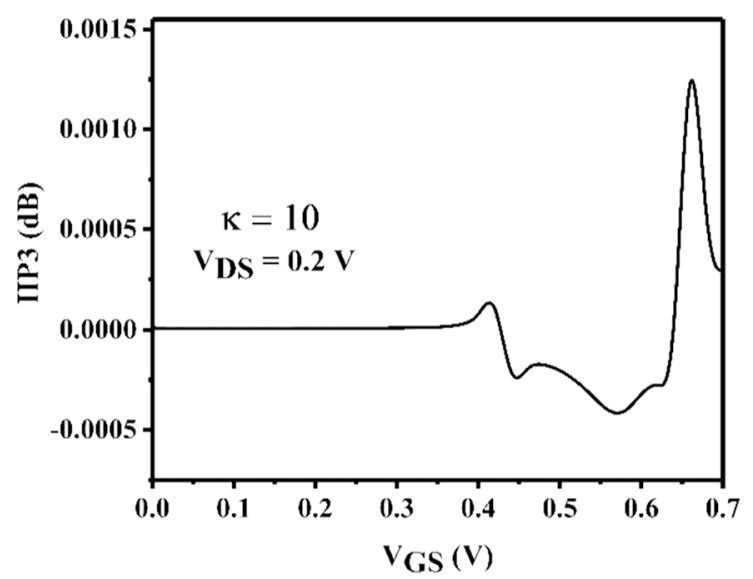

(c)

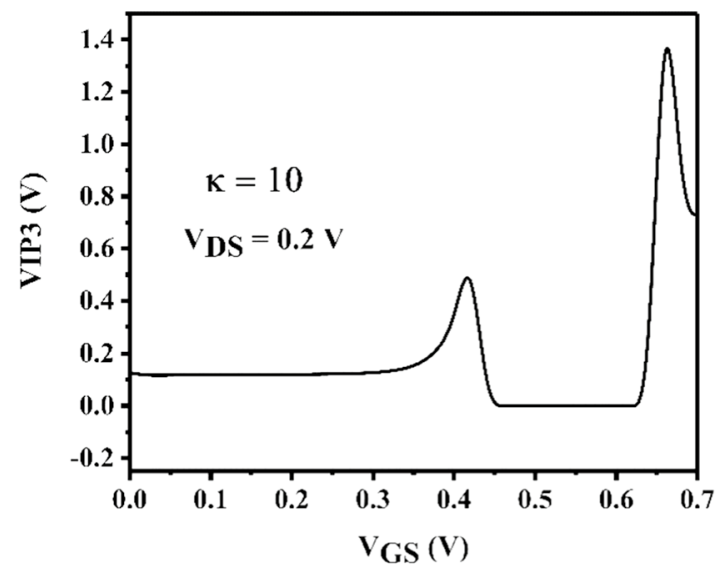

(b)

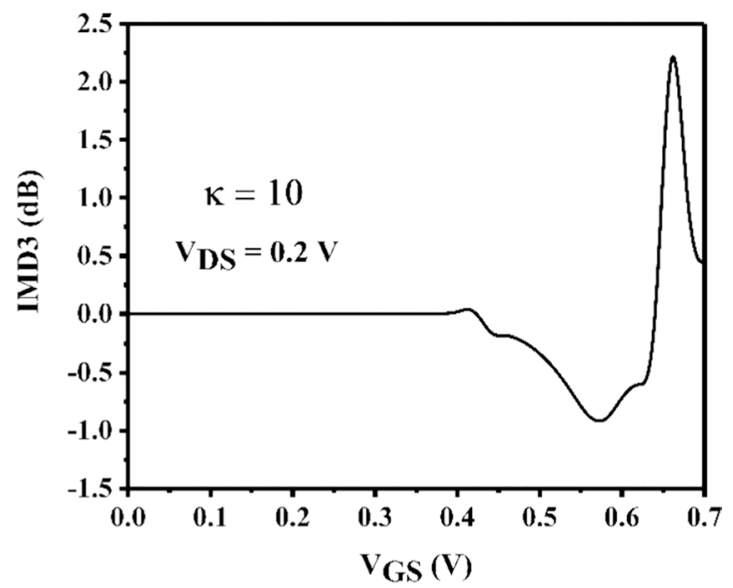

(d)

Fig. 9 a gm3, b VIP3, c IIP3, and d IMD3 profile of the proposed device with keratin $(k=10)$ at $V_{\mathrm{DS}}=0.2 \mathrm{~V}$

thermal noise characteristics, resulting in the improved noise immunity profile of the DM DT GE-MOSFET-based biosensor in the presence of biomolecules. Here, the minimum noise figure for a cavity filled with keratin $(K=10)$ is $1.8 \mathrm{~dB}$ at a high frequency of $100 \mathrm{GHz}$, thus it can be said that the proposed device is noise immune. Figure 10c illustrates another pivotal noise FOM, termed the optimum source impedance, which is very close to zero at higher frequencies in the case of an air-filled cavity but gradually increases with increasing dielectric constant of the biomolecule. This finding therefore indicates that the proposed device can exhibit very low noise in the presence of biomolecules and could represent an alternative for use in biosensing applications.

\section{Conclusions}

The electrical characteristics of the proposed device are analyzed in terms of the threshold voltage shift, switching or $I_{\mathrm{ON}} / I_{\mathrm{OFF}}$ ratio, surface potential, RF sensitivity, linearity, and noise FOMs. The results reveal that the inclusion of the ITO gate in the nanocavity significantly enhances the performance of the DM DT GE-MOSFET-based biosensor. Moreover, the proposed device exhibits a maximum $V_{\mathrm{th}}$ shift of nearly $100 \mathrm{mV}$ for $K=10$. Furthermore, the DM DT GE-MOSFET-based biosensor also exhibits an impressive sensitivity profile in terms of its RF sensing characteristics such as the transconductance gain and cutoff frequency. It 


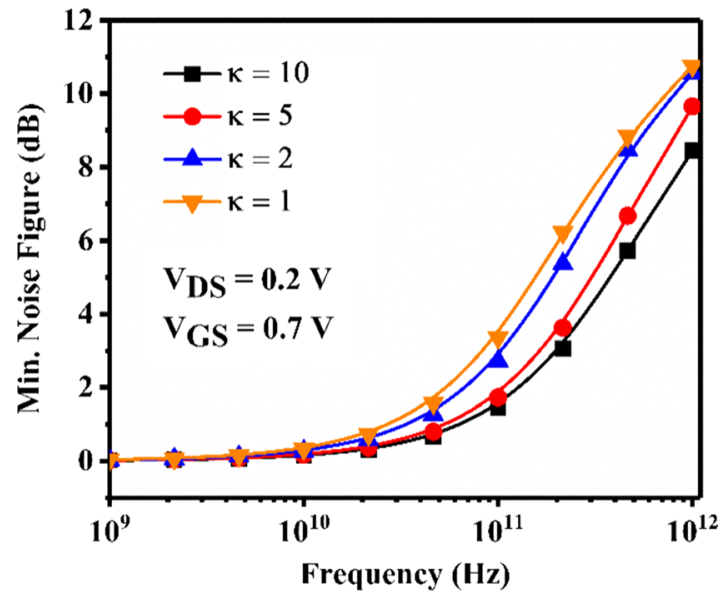

(a)

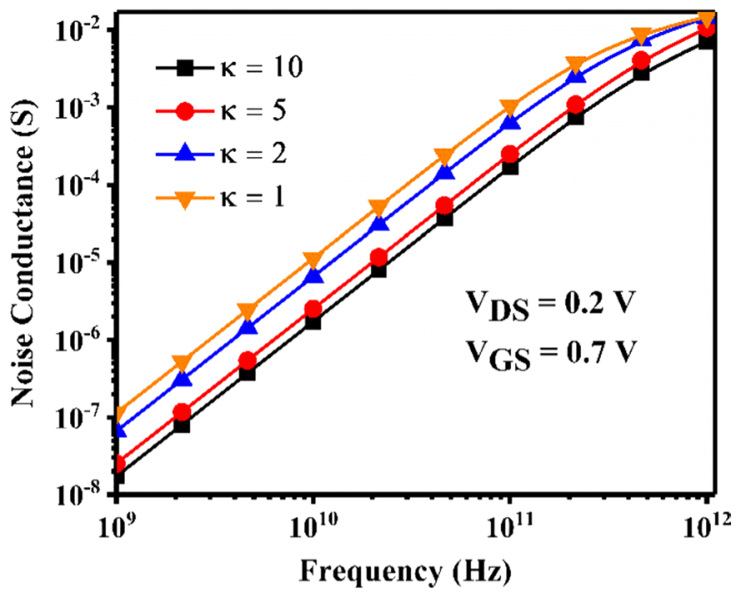

(b)

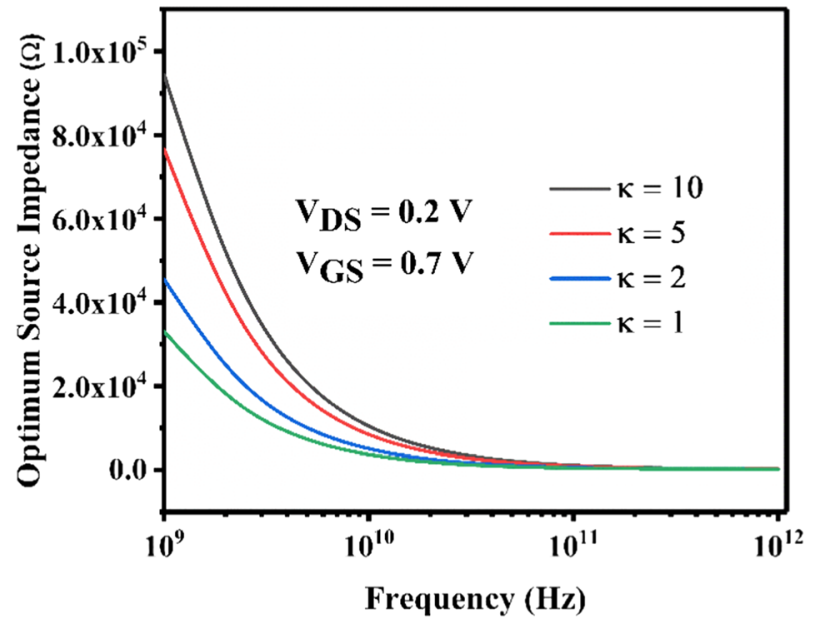

(c)

Fig. 10 a The minimum noise figure, $\mathbf{b}$ the noise conductance, and $\mathbf{c}$ the optimum source impedance profile of the proposed device for different biomolecules

is found that the highest sensitivity, observed in the case of keratin $(K=10)$, also corresponds to higher noise immunity at high frequency. Therefore, the DM DT GE-MOSFETbased biosensor could represent an emerging, highly sensitive and noise-immune device for the detection of biomolecules based on the dielectric-modulation technique at a low operating voltage.

Acknowledgements The authors of this manuscript gratefully acknowledge the Department of ETCE, Jadavpur University for support.

Data Availability Data will be made available on reasonable request.

\section{Declarations}

Conflict of interest The authors would like to state that they have no conflicts of interest.

\section{References}

1. Mukhopadhyay, S., Sen, D., Goswami, B., Sarkar, S. K.: Performance evaluation of dielectrically modulated extended gate single cavity InGaAs/Si HTFET based Label-free Biosensor considering non-ideal issues. In: IEEE Sens. J., https://doi.org/10.1109/JSEN. 2020.3033576.

2. Chen, X., Guo, Z., Yang, G.-M., Li, J., Li, M.-Q., Liu, J.-H., Huang, X.-J.: Electrical nanogap devices for biosensing. Mater. Today 13(11), 28-41 (2010)

3. Narang, R., Reddy, K.V.S., Saxena, M., Gupta, R.S., Gupta, M.: A Dielectric-modulated tunnel-FET-based biosensor for label-free detection: analytical modeling study and sensitivity analysis. IEEE Trans. Electron Devices 59(10), 2809-2817 (2012). https://doi. org/10.1109/TED.2012.2208115

4. Bergveld, P.: The development and application of FET-based biosensors. Biosensors 2(1), 15-33 (1986)

5. Wenga, G., Jacques, E., Salaun, A.-C., et al.: Step-gate polysilicon nanowires field effect transistor compatible with CMOS technology for label-free DNA biosensor. Biosens. Bioelectron. 40(1), 141-146 (2013) 
6. Guan, W., Duan, X., Reed, M.A.: Highly specific and sensitive nonenzymatic determination of uric acid in serum and urine by extended gate field effect transistor sensors. Biosens. Bioelectron. 51, 225-231 (2014)

7. Kanungo, S., Chattopadhyay, S., Gupta, P.S., Rahaman, H.: Comparative performance analysis of the dielectrically modulated full- gate and short-gate tunnel FET-based biosensors. IEEE Trans. Electron Devices 62(3), 994-1001 (2015). https:// doi.org/10.1109/TED.2015.2390774

8. Im, H., Huang, X.J., Gu, B., et al.: A dielectric-modulated fieldeffect transistor for biosensing. Nat. Nanotechnol. 2(7), 430-434 (2007)

9. Kim, C. H., Jung, C., Lee, K. B. et al.: Label-free DNA detection with a nanogap embedded complementary metal oxide semiconductor. Nanotechnology, 22(13), 135502-1-135502-5, (2011).

10. Gu, B., Park, T.J., Ahn, J.H., et al.: Nanogap field-effect transistor biosensors for electrical detection of avian influenza. Small 5(21), 2407-2412 (2009)

11. Kim, C.H., Jung, C., Park, H.G., et al.: Novel dielectric modulated field-effect transistor for label-free DNA detection. Biochip J. 2(2), 127-134 (2008)

12. Choi, J. M., Han, J. W., Choi, S. J. et al.: Analytical modeling of a nanogap-embedded FET for application as a biosensor. IEEE

13. Im, H., Huang, X.-J., Gu, B., Choi, Y.-K.: A dielectric-modulated field-effect transistor for biosensing. Nat. Nanotechnol. 2(7), 430 434 (2007)

14. Ajay, Gupta, M., Narang, R., Saxena, M.: Analysis of GaSb-InAs gate all around (GAA) p-i-n tunnel FET (TFET) for application as a biosensor. In: Proc. IEEE Int. Nanoelectron. Conf. (INEC), (2016), pp. 1-2.

15. Bibi, F. et al. A review: origins of the dielectric properties of proteins and potential development as bio-sensors. Sensors (Basel, Switzerland), 16(8): 1232. https://doi.org/10.3390/s16081232.

16. Goswami, R., Bhowmick, B.: Comparative analyses of circular gate TFET and heterojunction TFET for dielectric-modulated label free biosensing. IEEE Sens. J. 19(21), 9600-9609 (2019)
17. Talley, K., Ng, C., Shoppell, M., Kundrotas, P., Alexov, E.: On the electrostatic component of protein-protein binding free energy. PMC Biophys. https://doi.org/10.1186/1757-5036-1-2(2008).

18. Ajay, Narang, R., Saxena, M., Gupta, M.: Modeling of gate underlap junctionless double gate MOSFET as bio-sensor. Mater. Sci. Semiconduct. Process., 71, 240-251 (2017).

19. Chanda, M., Das, R., Kundu, A., Sarkar, C.K.: Analytical modeling of label free biosensor using charge plasma based gate underlap dielectric modulated MOSFET. Superlattices Microstruct. 104, 451-460 (2017)

20. Chakraborty, A., Sarkar, A.: Analytical modeling and sensitivity analysis of dielectric-modulated junctionless gate stack surrounding gate MOSFET (JLGSSRG) for application as biosensor. J. Comput. Electron. 16(3), 556-567 (2017)

21. Ajay, Narang, R., Saxena, M., Gupta, M.: Modeling and simulation investigation of sensitivity of symmetric split gate junctionless FET for biosensing application. IEEE Sens. J., 17(15), 4853-4861 (2017)

22. ATLAS Device Simulation Software, Silvaco Int., Santa Clara, CA, USA, (2014).

23. Appenzeller, J., Martel, R., Avouris, P., Knoch, J., Scholvin, J., del Alamo, J.A., Rice, P., Solomon, P.: Sub-40 nm SOI V-groove n-MOSFETs. IEEE Electron Device Lett. 23(2), 100-102 (2002)

24. Kannan, N., Kumar, M.J.: Dielectric-modulated impact-ionization MOS transistor as a label-free biosensor. IEEE Electron Dev. Lett. 34(12), 1575-1577 (2013)

25. Bhattacharyya, A., Chanda, M., De, D.: Analysis of NoiseImmune Dopingless Heterojunction Bio-TFET considering partial hybridization issue. IEEE Trans. Nanotechnol. 19, 769-777 (2020). https://doi.org/10.1109/TNANO.2020.3033966

26. Kumar, A., Tripathi, M.M., Chaujar, R.: Ultralow-power dielectric-modulated nanogap-embedded sub-20-nm TGRC-MOSFET for biosensing applications. J. Comput. Electron. 17, 1807-1815 (2018). https://doi.org/10.1007/s10825-018-1237-2

Publisher's Note Springer Nature remains neutral with regard to jurisdictional claims in published maps and institutional affiliations. 